\title{
Direction of Arrival (DoA) Estimation for a Switched-Beam DS-CDMA System using Neural Networks
}

UDK 621.396.67:004.032.26

IFAC $3.1 .1 ; 4.3 .2$

Original scientific paper

A Neural Network (NN) methodology is presented for the Direction of Arrival (DoA) estimation of the desired mobile user, namely the Signal of Interest (SoI), in the asynchronous Direct Sequence Code Division Multiple Access (DS-CDMA) scheme. In DS-CDMA the SoI DoA should be estimated in the presence of a group of interfering users that constitute the Multiple Access Interference (MAI). In this paper a NN algorithm follows the MAI suppression done by the code-matched filters, to achieve the DoA finding goal for a Switched-Beam System (SBS). The proposed method is developed, while DoA estimation simulation tests show accurate results for a varying number of mobile users.

Key words: direction of arrival, DS-CDMA, neural networks, switched-beam system

\section{INTRODUCTION}

Direct Sequence Code Division Multiple Access (DS-CDMA) is a widely used air interface in modern wireless communications. Unlike Time Division Multiple Access (TDMA) and Frequency Division Multiple Access (FDMA), in DS-CDMA the mobile users communicate simultaneously at the same frequency band. Each user is assigned a unique code sequence that encodes the transmitted data signal, and enables its separation and recovery at the receiver by code-matched filters containing the corresponding codeword [1]. The codewords are orthogonal to each other, however the uplink normally operates asynchronously and the orthogonality breaks down. Therefore, the Signal of Interest (SoI) interferes throughout the code-matched filter with the other users' signals that form the Multiple Access Interference (MAI). In [2] it is shown that in this case the MAI tends toward the real Gaussian distribution with zero mean. In the same work the MAI suppression followed by the subspace algorithm MUSIC [3] has been employed. In this way, the DoA estimation scenario of signals impinging on the antenna array of a DS-CDMA system, is reduced to the localization of a single (desired) source in a noise environment. Parallel such implementations overcome the restriction of the conventional application of subspace algorithms, which is that the number of antenna elements must exceed the number of the incoming signals [4]. In [5] the Matrix Pencil (MP) method has been extended to the CDMA case, having the advantage of achieving successful DoA estimation by using only a single snapshot [6]. The MUSIC, the MP and other widespread DoA finding algorithms are in principle designed for Adaptive Array Systems (AAS), involving computationally intensive processes, such as eigenvalue decomposition and signal auto-correlation matrix calculations.

The market seems to slow the adoption of adaptive antenna beamforming techniques mainly due to the implementation complexity of such systems [7]. In [8] a simple and accurate Neural Network (NN) methodology has been developed for the DoA estimation of a Switched-Beam System (SBS), requiring only power measurements at the output of the system. Switched-beam systems are quite popular as they offer many of the advantages of the fully adaptive ones at less expense and complexity. In this work the statistical properties of the code sequences and data bits are used, to show how in the asynchronous DS-CDMA system the SoI power is boosted over the power of the other signals. The NN methodology for the SBS [8] is extended to the DS-CDMA case, taking advantage of the SoI processing gain by applying the NN algorithm after the code-matched filter.

The paper is outlined as follows: In Section 2 the signal power model for the DS-CDMA Switched-Beam Antenna System (SBAS) is developed. In Section 3 the NN methodology for the DoA es- 
timation is described, followed by simulation tests in Section 4. Finally, Section 5 concludes the paper. An Appendix is given where useful statistical properties are derived.

\section{SIGNAL POWER MODEL OF THE SYSTEM}

\subsection{Transmitted signals}

The uplink of a DS-CDMA system with $N$ mobile users is considered. Each transmitted data bit $b_{l}(t) \in\{-1,1\}$ of the $l^{\text {th }}$ user is directly multiplied by a unique codeword of $Q$ bits, called chips. Let the data bit period be $T_{b}$, while the chip period is $T_{c}=T_{b} / Q$. The vector $\mathbf{c}_{l}(t)$ denotes the codeword of the $l^{\text {th }}$ user, which is orthogonal to the code sequences assigned to the other users:

$$
\mathbf{c}_{l}(t)=\left[c_{l 1} c_{l 2} \ldots c_{l Q}\right]^{T} \quad l=1, \ldots, n, \ldots, N .
$$

The orthogonality breaks down in the asynchronous DS-CDMA system, thus each element of the code vectors can be modeled as an independent, identically distributed (iid) binary $( \pm 1)$ random variable (RV) with zero mean [2]. The transmitted baseband signals during a bit period can be written as:

$$
\mathbf{s}=\sum_{l=1}^{N} v_{l} b_{l} \mathbf{c}_{l}
$$

where $v_{l}$ is the signal amplitude of the $l^{\text {th }}$ user. Since the chip rate of the code signals is greater than the bit rate of the information signals, the transmitted signals are spread over a large bandwidth. This spreading property places DS-CDMA among the Spread Spectrum Multiple Access (SSMA) techniques.

\subsection{The Switched-Beam Antenna System}

The transmitted signals are received by a SBAS (Figure 1), which relies on an $8 \times 8$ Butler Matrix (BM) that feeds a uniform linear array (ULA) of $M=8, \lambda / 2$ spaced, rectangular microstrip patches. The patches are structured on a single layer dielectric substrate. The BM is a common used fixed beamforming network consisted of hybrid couplers, phase shifters, and crossovers [9], whose input ports are connected to a switching network that performs the beam switching. Each input port $i=$ $=1,2, \ldots 8$ corresponds to an array weight vector $\mathrm{w}_{i}=\left[w_{i 1}, w_{i 2}, \ldots w_{i M}\right]^{T}$ that produces a different fixed phase difference between the antenna elements. The system is designed to give a set of eight predefined orthogonal beams that cover an angular sector of $120^{\circ}$. The total field pattern for the $i^{\text {th }}$ beam is given by:

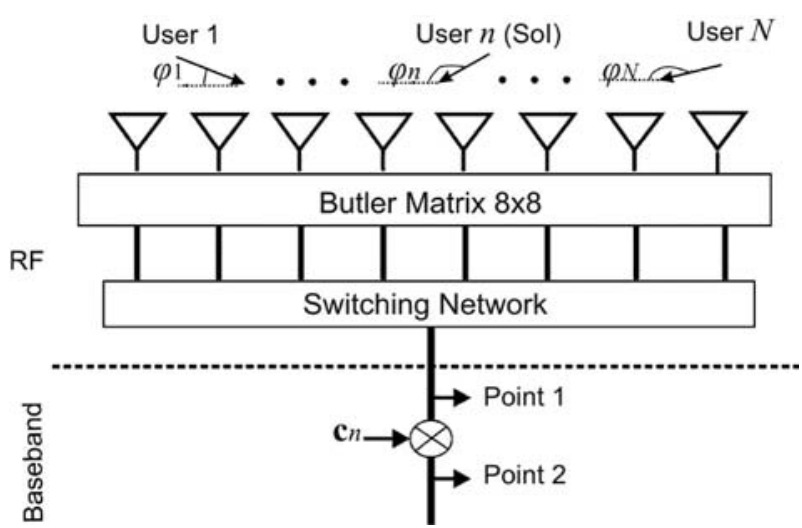

Fig. 1 Switched-beam DS-CDMA system used for DoA estimation

$$
\overrightarrow{\mathrm{W}}_{i}(\phi)=\mathbf{w}_{i}^{H} \boldsymbol{a}(\phi) \overrightarrow{\mathrm{g}}(\phi)
$$

where $\mathbf{a}(\phi)=\left[1 e^{j \pi \sin \phi} \ldots e^{j(M-1) \pi \sin \phi}\right]^{T}$ is the steering vector in the azimuthal direction $\phi$ and $\vec{g}(\phi)$ is the vector of the field pattern of the microstrip patch. The power pattern $P_{i}(\phi)=\left|\vec{W}_{i}(\phi)\right|^{2}$ is calculated at an angle step of $\Delta \phi=0.5^{\circ}$ and its plot is given in Figure 2.

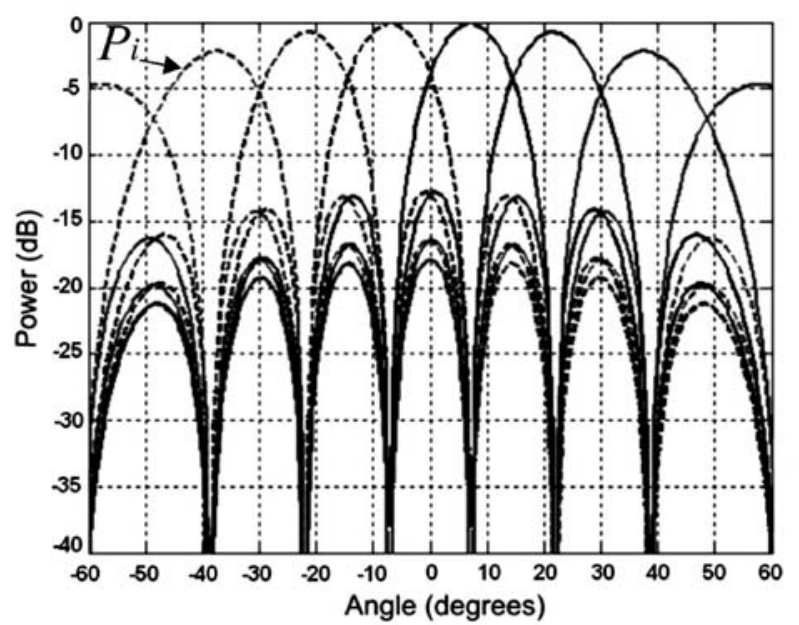

Fig. 2 Calculated power pattern for each beam $P_{i}$ of the SBS

\subsection{Received signal power}

The transmitted signals from the $N$ mobile users impinge on the proposed SBAS from different random azimuthal directions $\phi_{1}, \ldots, \phi_{n}, \ldots \phi_{N}$ within an $120^{\circ}$ angle sector:

$$
-60^{\circ} \leq \phi_{l} \leq 60^{\circ}, l=1, \ldots, n, \ldots, N .
$$

It is considered that the received signal from each mobile arrives from a single angle. Let the 
SoI come from the $n^{\text {th }}$ angle, while the other angles correspond to the MAI composing an angle of arrival (AoA) vector $\varphi_{I}=\left(\phi_{1}, \phi_{2}, \ldots \phi_{N}\right), \phi_{n} \notin \boldsymbol{\varphi}_{I}$. If beam switching takes place, the total received signal before the filter (point 1 in Figure 1), concerning the $i^{\text {th }}$ beam and the $k^{\text {th }}$ bit of each user, is expressed by:

$$
\begin{gathered}
\mathbf{Y}_{1}^{i}(k)=h_{n} b_{n}(k)\left|\overrightarrow{\mathrm{W}}_{i}\left(\phi_{n}\right)\right| \mathbf{c}_{n}^{T}(k)+ \\
+\sum_{l=1, l \neq n}^{N} h_{l} b_{l}(k)\left|\overrightarrow{\mathrm{W}}_{i}\left(\phi_{l}\right)\right| \mathbf{c}_{l}^{T}(k)= \\
=\mathbf{Y}_{1 S}^{i}(k)+\mathbf{Y}_{1 I}^{i}(k) .
\end{gathered}
$$

In this study only the MAI is considered and not any other source of noise. To simplify formulation the symbols for the $k^{\text {th }}$ bit and the $i^{\text {th }}$ beam are omitted and the corresponding mean output power is given by:

$$
P_{1 \mathrm{~T}}=E\left\{\mathbf{Y}_{1} \mathbf{Y}_{1}^{H}\right\}=E\left\{\left(\mathbf{Y}_{1 S}+\mathbf{Y}_{1 I}\right)\left(\mathbf{Y}_{1 S}^{H}+\mathbf{Y}_{1 I}^{H}\right)\right\}
$$

where $E\{\cdot\}$ denotes the expectation operator.

In the Appendix the statistical properties of the code vector and the data bit are derived. Using these properties it can be proved that:

$$
\begin{gathered}
E\left\{\mathbf{Y}_{1 S} \mathbf{Y}_{1 S}^{H}\right\}=Q h_{n}{ }^{2} P\left(\phi_{n}\right) \\
E\left\{\mathbf{Y}_{1 S} \mathbf{Y}_{1 I}^{H}\right\}=E\left\{\mathbf{Y}_{1 I} \mathbf{Y}_{1 S}^{H}\right\}=0 \\
E\left\{\mathbf{Y}_{1 I} \mathbf{Y}_{1 I}^{H}\right\}=Q \sum_{l=1, l \neq n}^{N} h_{l}{ }^{2} P\left(\phi_{l}\right) .
\end{gathered}
$$

Thus, the output power for the $i^{\text {th }}$ beam before the code-matched filter is given by:

$$
P_{1 \mathrm{~T}}^{i}=\underbrace{Q h_{n}{ }^{2} P_{i}\left(\phi_{n}\right)}_{\text {SoI }}+\underbrace{Q \sum_{l=1, l \neq n}^{N} h_{l}^{2} P_{i}\left(\phi_{l}\right)}_{\text {MAI }} .
$$

In order to pick out the SoI, a code-matched filter containing the corresponding codeword $\mathbf{c}_{n}$ is applied to the received signal $\mathbf{Y}_{1}$. At the filter output (point 2 in Figure 1) there is:

$$
\begin{gathered}
\mathbf{Y}_{2}=\mathbf{Y}_{1} \mathbf{c}_{n}=h_{n} b_{n}\left|\overrightarrow{\mathrm{W}}_{i}\left(\phi_{n}\right)\right| \underbrace{\mathbf{c}_{n}^{T} \mathbf{c}_{n}}_{Q}+ \\
+\sum_{l=1, l \neq n}^{N} h_{l} b_{l}\left|\overrightarrow{\mathrm{W}}_{i}\left(\phi_{l}\right)\right| \mathbf{c}_{l}^{T} \mathbf{c}_{n}=\mathbf{Y}_{2 S}+\mathbf{Y}_{2 I} \\
\left.P_{2 \mathrm{~T}}=E\left\{\mathbf{Y}_{2} \mathbf{Y}_{2}{ }^{H}\right\}=E\left\{\mathbf{Y}_{2 S}+\mathbf{Y}_{2 I}\right)\left(\mathbf{Y}_{2 S}^{H}+\mathbf{Y}_{2 I}^{H}\right)\right\} .
\end{gathered}
$$

Proceeding with each term of the above product and according to the Appendix:

$$
\begin{gathered}
E\left\{\mathbf{Y}_{2 S} \mathbf{Y}_{2 S}^{H}\right\}=Q^{2} h_{n}{ }^{2} P\left(\phi_{n}\right) \\
E\left\{\mathbf{Y}_{2 S} \mathbf{Y}_{2 I}^{H}\right\}=E\left\{\mathbf{Y}_{2 I} \mathbf{Y}_{2 S}^{H}\right\}=0 \\
E\left\{\mathbf{Y}_{2 I} \mathbf{Y}_{2 I}^{H}\right\}=Q \sum_{l=1, l \neq n}^{N} h_{l}{ }^{2} P\left(\phi_{l}\right) .
\end{gathered}
$$

Therefore the output power for the $i^{\text {th }}$ beam after the code-matched filter is given by:

$$
P_{2 \mathrm{~T}}^{i}=\underbrace{Q^{2} h_{n}{ }^{2} P_{i}\left(\phi_{n}\right)}_{\text {SoI }}+\underbrace{Q \sum_{l=1, l \neq n}^{N} h_{l}^{2} P_{i}\left(\phi_{l}\right)}_{\text {MAI }} .
$$

From (10) and (16) it is concluded that the desired signal is despread and boosted by a factor of $Q$, called the processing gain of the DS-CDMA system. The MAI signals remain spread over a large bandwidth statistically producing a noise background. The aim is to extract the DoA of the desired user from this interference environment.

\section{NEURAL NETWORK DoA ESTIMATION}

\subsection{Methodology}

In [8] a generic NN-DoA estimation method has been developed for a SBS having two basic requirements: a) power control, and b) a priori knowledge of the number of the simultaneous incoming signals. The proposed methodology can be successfully extended in a DS-CDMA scheme, as both requirements are de facto fulfilled: a) power control application to the mobile users is an inherent feature of DS-CDMA, and b) one single DoA has to be estimated at a time.

Consider the DoA estimation scenario described in subsection 2.3. Due to power control all signals are received at the base station at the same mean power level, which is considered to be unity $\left(h_{1}=\right.$ $=1, l=1,2, \ldots, N)$. If beam switching takes place (16) gives a system of equations expressing the total power received for each beam after the despreading:

$$
\begin{aligned}
& P_{2 \mathrm{~T}}^{1}=Q^{2} P_{1}\left(\phi_{n}\right)+Q \sum_{l=1, l \neq n}^{N} P_{1}\left(\phi_{l}\right) \\
& P_{2 \mathrm{~T}}^{2}=Q^{2} P_{2}\left(\phi_{n}\right)+Q \sum_{l=1, l \neq n}^{N} P_{2}\left(\phi_{l}\right) \\
& P_{2 \mathrm{~T}}^{8}=Q^{2} P_{8}\left(\phi_{n}\right)+Q \sum_{l=1, l \neq n}^{N} P_{8}\left(\phi_{l}\right) .
\end{aligned}
$$


Equation (17) shows that for a given processing gain $Q$, the total power $P_{2 \mathrm{~T}}^{i}$ measured for the $i^{\text {th }}$ beam depends on the AoA of the incoming signals and the radiation pattern of the beam. Therefore a mapping is established between the power vector $\mathbf{P}_{2}=\left(P_{2 \mathrm{~T}}^{1}, P_{2 \mathrm{~T}}^{2}, \ldots, P_{2 \mathrm{~T}}^{8}\right)$ and the corresponding angle $\phi_{n}$ and angle vector $\varphi_{I}$. A large number of such mappings should be created to train the DoA estimation NN through a supervised learning procedure.

\subsection{Neural Networks}

$M$ sets of $N$ AoA are randomly generated. $N-1$ angles of each set compose the MAI AoA vector and an angle $\boldsymbol{\varphi}_{n m}$ is the SoI AoA. The index $m$ denotes the $m^{\text {th }}$ angle vector and the $m^{\text {th }}$ SoI angle. The random angle values are equal to integer multiples of $\Delta \phi=0.5^{\circ}$ within the sector angular range. Equation (17) gives the corresponding power vectors $\mathbf{P}_{2 m}$ and a collection of randomly created pairs $\left(\phi_{n m}, \overline{\mathbf{P}}_{2 m}^{m}\right)$ is generated that is used as the training set for the NN. The same NN (for a given processing gain) should be used to perform DoA estimation for a varying number of interferers. Thus, the value of $N$ for each pair of the training set varies from 2 to 40 users (i.e. 1 to 39 interferers).

A Multilayer Perceptron (MLP) NN is used [10] composed by: 1) an input layer of eight nodes fed by the vectors $\mathbf{P}_{2 m}, 2$ ) an output layer of one node that gives the corresponding DoA $\phi_{n m}$, and 3) two hidden layers. The activation function of the hidden layers is the hyperbolic tangent function, whereas of the output layer is linear. The number of pairs composing the training set is $M=5000$. It is noted that this number is much smaller than the number of all possible $\varphi_{I m}$ and $\phi_{n m}$. This shows the effectiveness and the generalization capabilities of a properly trained NN. Generally, the number of training pairs should be large enough to favour the generalization, but also upper limited in order to avoid a quick saturation due to over-training.

The NN training has been performed to MATLAB using the optimisation algorithm Levenberg-Marquardt (LM) [11]. The LM algorithm provides a relatively fast numerical solution to the minimization of the performance function, which is the Mean Square Error (MSE). The MSE during the training is defined as the averaged squared error between the network outputs and the target outputs. The training stops when the performance goal is achieved or the MSE minimization reaches a plateau. Two NNs, one for $Q=256$ and another for $Q=128$ have been trained. These values of processing gain are commonly used in UMTS communication channels, as specified in $3 \mathrm{GPP}$ technical specifications [12].

\section{SIMULATIONS}

Since the training of the $\mathrm{NN}$ is over, the DoA estimation procedure is summed up to the following four steps: a) Simultaneous arrival of $N$ signals. b) Beam switching and power measurement for each beam at the output of the code-matched filter. c) Feeding of the trained NN with the measured power vector. d) NN calculation of the SoI

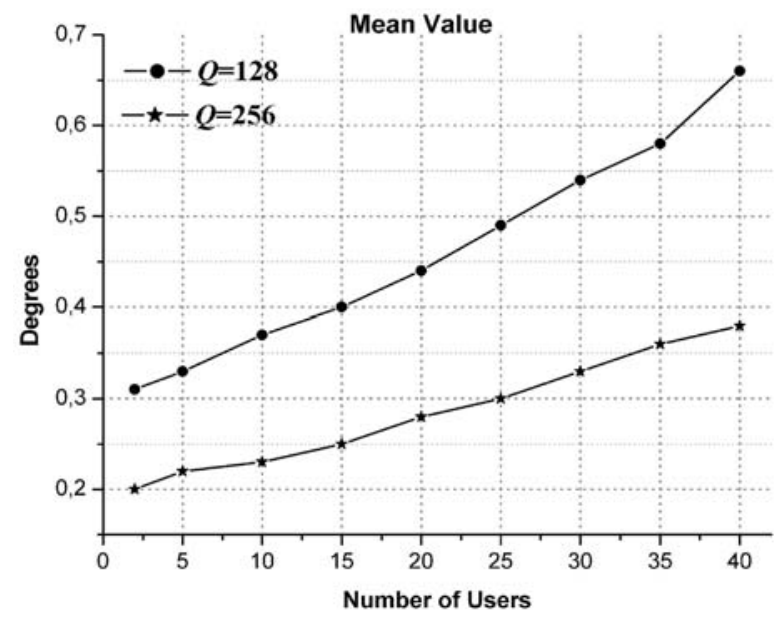

Fig. 3 Mean value of the absolute difference between the expected and estimated DoA, 4000 simulation tests with processing gain $Q=128$ and $Q=256$

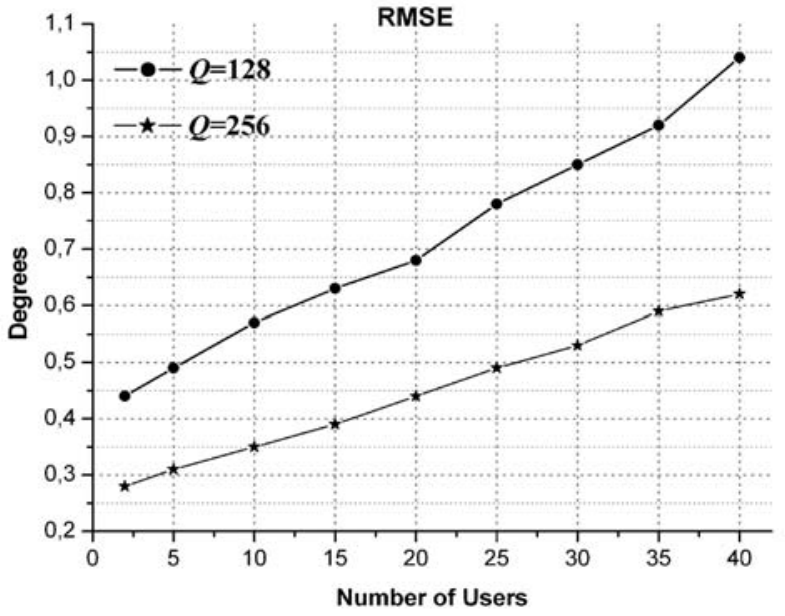

Fig. 4 RMSE of the absolute difference between the expected and estimated DoA, 4000 simulation tests with processing gain $Q=128$ and $Q=256$ 
DoA. In order to evaluate the proposed approach, the whole procedure has been simulated in MATLAB. Random sets of $\phi_{n}$ and $\varphi_{I}$ are generated, simulating the simultaneous incidence of one SoI and $\mathrm{N}-1 \mathrm{MAI}$ signals. These AoA are inserted into (17) producing the corresponding power vectors $\mathbf{P}_{2}$ that simulate the power that would be measured in a real DoA estimation problem. The power vectors are then fed to the NN that instantly calculates the DoA of the desired user. The accuracy of the technique is tested by comparing the estimated SoI DoA $\phi_{n}^{\text {est }}$ with the expected DoA $\phi_{n}$.
DoA estimation of the desired source has been tested for 4000 independent simulation runs, at the presence of a varying number of interferers from 1 to 39 . The quantity

$$
\Delta \phi_{i}^{e s t}=\left|\phi_{i}^{e s t}-\phi_{n i}\right|, i=1,2, \ldots, 4000
$$

has been statistically processed and the results are presented in Figures 3 and 4. In these diagrams, the mean value and the Root Mean Square Error (RMSE) of $\Delta \phi_{n}^{e s t}$ are plotted versus the number of mobile users for the two values of processing gain

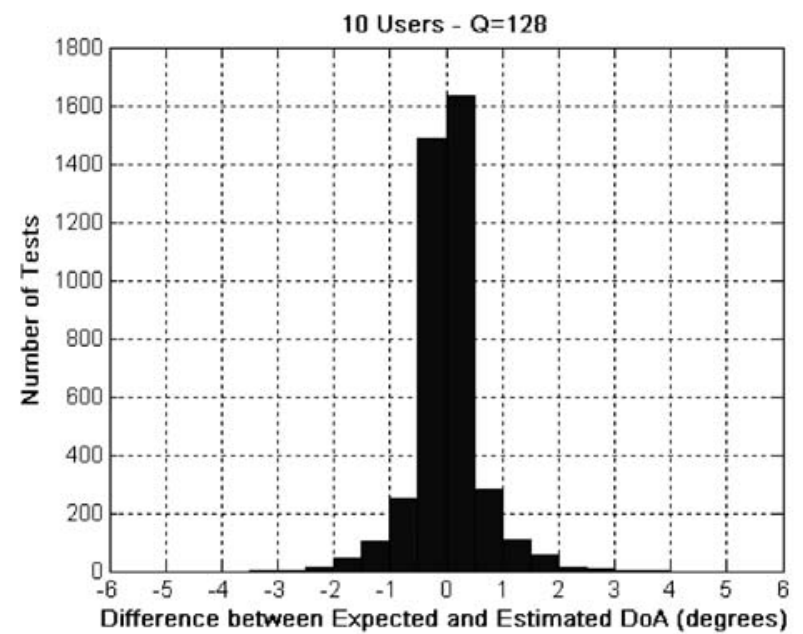

a)

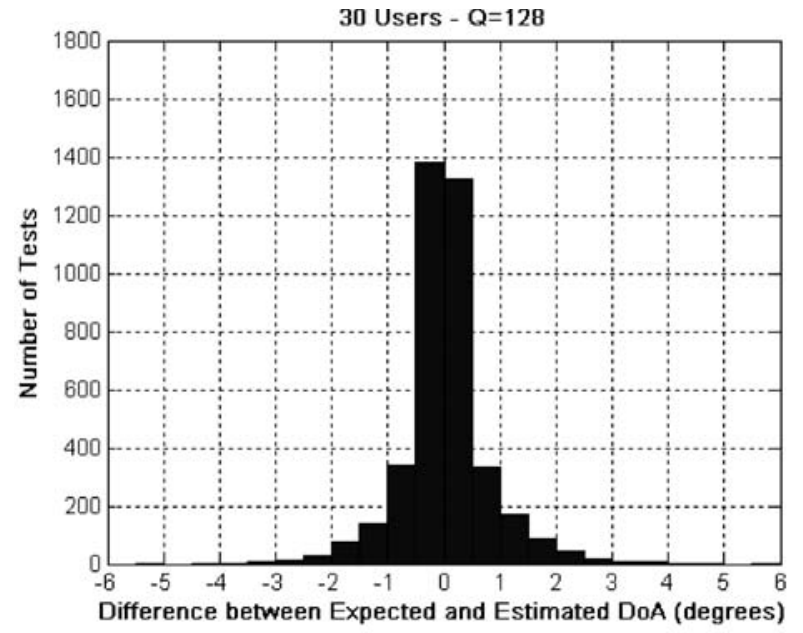

b)

Fig. 5 Distribution of difference between the expected and estimated DoA, 4000 simulation tests with processing gain $Q=128$ for, a) 10 users, and b) 30 users

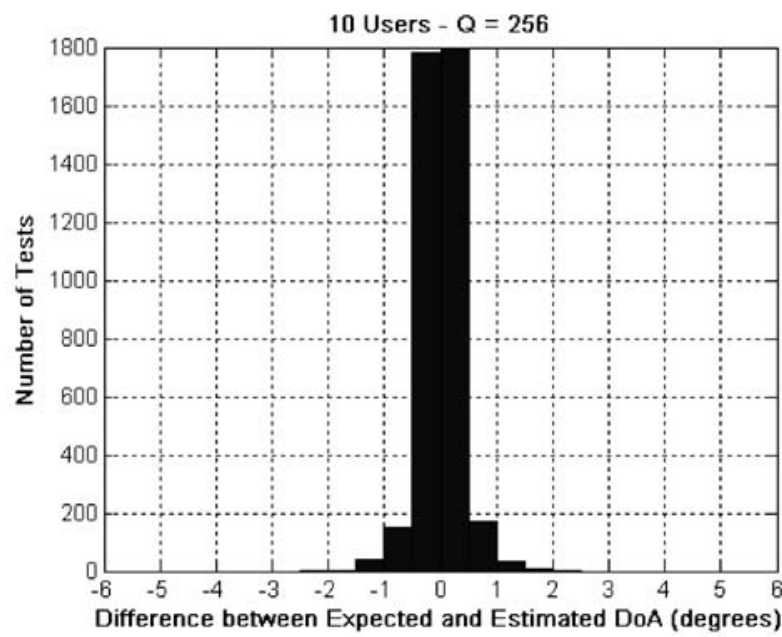

a)

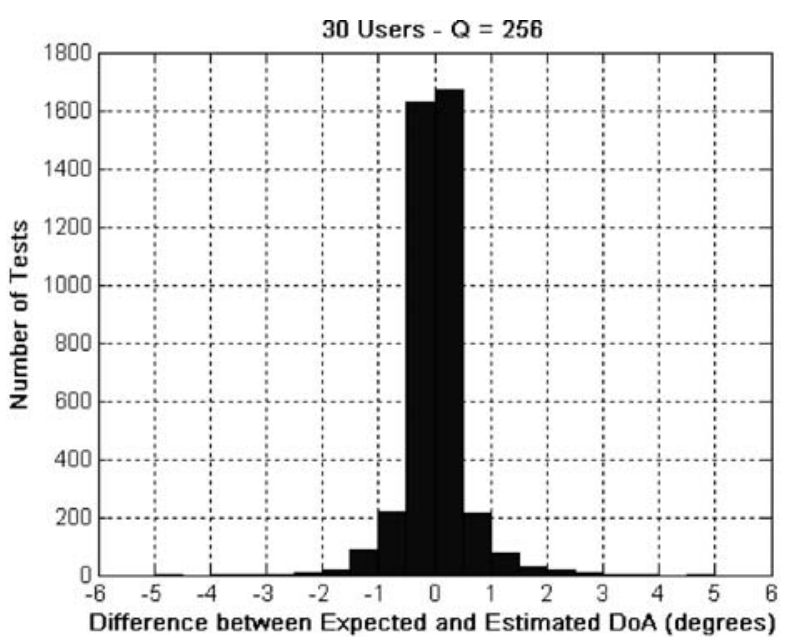

b)

Fig. 6 Distribution of difference between the expected and estimated DoA, 4000 simulation tests with processing gain $Q=256$ for, a) 10 users, and b) 30 users 
$Q=128$ and $Q=256$. The obvious conclusion derived is that the method performs better for the bigger value of processing gain, as the MAI suppression is deeper in that case and the SoI extraction is much easier. Moreover, it is observed that the slope in the transition from 35 to 40 users is quite steeper for $Q=128$ than for $Q=256$. This shows that in the case of the small processing gain the $\mathrm{NN}$ is less effective at the upper limits of the users training range. However, even in this extreme case of 40 users and $Q=128$ the results are quite satisfactory, since the RMSE and the mean value of $\Delta \phi_{n}^{e s t}$ are about 1 and 0.7 degrees respectively. Finally it is noticed that although the RMSE remains in low levels, its increase is steeper compared to that of the mean value. This indicates that the dispersion of the results increases with the number of interferers. Thus, the NN training could be further improved in order to demonstrate a more stable behavior in the whole range of users.

Some diagrams concerning a specific number of users follow the above aggregate results. In Figures 5 and 6 , the distribution of difference (per 0.5 degrees) between the expected and the estimated DoA is given for the cases of 10 and 30 users. In these diagrams the impact of the test parameters (processing gain-number of interferers) to the estimation's accuracy is illustrated more visually. A useful observation is that the distributions are nearly symmetric around zero, indicating almost unbiased estimation. Additionally, for all cases, only a few estimation differences lie outside the interval of $[-2,2]$ degrees, with the majority of them concentrated within the interval of $[-0.5,0.5]$ degrees.

\section{CONCLUSIONS}

A neuro-computational methodology has been presented for the DoA estimation of a SBS operating in the asynchronous DS-CDMA scheme. The processing gain obtained through the corresponding code-matched filter has been exploited, in order to accomplish DoA estimation for the desired user at the presence of the MAI. Compared to other similar extensions to DS-CDMA $[2,5]$ the present approach has some special features. First of all it is designed for a SBS. Additionally, it relies on power measurements instead of the intensive signal processing required for the popular subspace algorithms (e.g. MUSIC). Finally, neural network techniques are employed. The above characteristics result to a less expensive and complex method compared to the adaptive beamforming ones.
Extended simulation tests have been performed and their results are presented and discussed. The effect of the processing gain and interferers' number to the performance of the method has been studied. Although it seems that the results could be further improved, the DoA estimation is accurate for a big range of simultaneous users. Even for the case of 40 users and processing gain 128, the DoA of the desired source is estimated with a RMSE of about one degree. This value is quite satisfactory for a switched-beam implementation, so as far as DoA estimation is concerned, a bigger value of processing gain may not be necessary.

In this paper the proposed methodology considers only the incoming signals that are subject to power control, namely the main paths. However, it could be applied at the presence of MAI that includes multipath signals. In DS-CDMA, if a delayed version of a signal is received outside the time interval $\left[-T_{c}, T_{c}\right]$, it will be treated as interference with a small contribution to the noise background. Therefore, the multipath signals do not deteriorate the performance of the DoA estimation procedure. However, their information and power should be exploited and combined properly in order to enhance the extraction of the desired signal.

\section{APPENDIX}

\section{Statistical properties of random variables}

Each element of the $l^{\text {th }}$ code vector $c_{l i}$ and each data bit of the $l^{\text {th }}$ user are iid random binary $( \pm 1)$ variables. The following statistical properties are obtained:

$$
\begin{gathered}
E\left\{c_{l i}\right\}=0, E\left\{c_{l i}^{2}\right\}=1, \quad l=1, \ldots, N \quad i=1, \ldots, Q \\
E\left\{c_{l i} c_{j n}\right\}=0, \quad \text { for } \quad l \neq j \text { or } i \neq n \\
E\left\{b_{l}\right\}=0, l=1, \ldots, N \\
E\left\{b_{l} b_{n}\right\}= \begin{cases}1, & \text { for } l=n \\
0, & \text { for } l \neq n\end{cases}
\end{gathered}
$$

Let $r_{l n}$ symbolize the correlation between the $l^{\text {th }}$ and the $n^{\text {th }}$ code vectors during the same bit interval:

$$
r_{l n}=\mathbf{c}_{l}^{T} \mathbf{c}_{n}=\sum_{i=1}^{Q} c_{l i} c_{n i} .
$$

In general the variance of a RV $x$ is given by [13]:

$$
\sigma^{2}=E\left\{x^{2}\right\}-[E\{x\}]^{2} .
$$


The product $c_{l i} c_{n i}$ is a RV with zero mean and unit variance. Based on the Central Limit Theorem [13], $r_{l n}$ has a probability distribution that tends to a normal or Gaussian curve as $Q \rightarrow \infty$.

According to (18), (19) and (22) there is:

$$
\begin{aligned}
& E\left\{r_{l n}\right\}=0 \\
& E\left\{r_{l n}^{2}\right\}=Q \\
& E\left\{r_{l n} r_{j n}\right\}=0 \text { for } l \neq j
\end{aligned}
$$

and from (23) $r_{l n}$ approaches a RV with zero mean and variance $Q$.

\section{ACKNOWLEDGEMENT}

This work is implemented in the framework of Measure 8.3 through the O.P. Competitiveness 3rd Community Support Programme and is co-funded by: $75 \%$ of the Public Expenditure from the European Union-European Social Fund, $25 \%$ of the Public Expenditure from the Hellenic State-Ministry of Development-General Secretariat for Research and Technology, and Private Sector (INTRACOM SA).

\section{REFERENCES}

[1] T. Ojanpera, R. Prasad, Wideband CDMA for Third Generation Mobile Communications. Artech House, Boston-London, 1998.

[2] C.-T. Chiang, A.-C. Chang, DoA Estimation in the Asynchronous DS-CDMA System. IEEE Transactions on Antennas and Propagation, vol. 51, no. 1, pp. 40-47, January 2003.

[3] R. O. Schmidt, Multiple Emitter Location and Signal Parameter Estimation. IEEE Transactions on
Antennas and Propagation, vol. 34, no. 3, pp. 276280, March 1986.

[4] L. C. Godara, Application of Antenna Arrays to Mobile Communications, part II: Beam-Forming and Direction-of-Arrival Considerations. Proc. IEEE, vol. 85, pp. 1195-1245, August 1997.

[5] C. K. E. Lau, R. S. Adve, T. K. Sarkar, Combined CDMA and Matrix Pencil Direction of Arrival Estimation. IEEE Vehicular Technology Conference, vol. 1, pp. 496-499, Fall, 2002.

[6] J. Koh, T. K. Sarkar, High Resolution DoA Estimation Using Matrix Pencil. IEEE Antennas and Propagation Society International Symposium, vol. 1, pp. 423-426, June 2004.

[7] F. Rayal, Why Have Smart Antennas Not Yet Gained Traction with Wireless Network Operators? IEEE Antennas and Propagation Magazine, vol. 47, no. 6, pp. 124-126, December 2005.

[8] K. A. Gotsis, E. G. Vaitsopoulos, K. Siakavara, J. N. Sahalos, Multiple Signal Direction of Arrival (DoA) Estimation for a Switched-beam System Using Neural Networks. Progress In Electromagnetics Research Symposium. pp. 420-424, Prague, Czech Republic, August 2007.

[9] J. Butler, R. Lowe, BeamForming Matrix Simplifies Design of Electronically Scanned Antennas. Electronic Design, Apr. 1961.

[10] C. Christodoulou, M. Georgiopoulos, Applications of Neural Networks in Electromagnetics. Artech House, Boston-London, 2001.

[11] H. Demuth, M. Beale, Neural Network Toolbox for Use With MATLAB, User's Guide (Fifth Edition). The Math Works, Inc., 1998.

[12] ..., 3G TS 25.213 V4.3.0 (2002-06), Spreading and Modulation (FDD).

[13] A. Papoulis, Probability, Random Variables, and Stochastic Processes. McGraw-Hill, 1984

Procjena smjera dolaska vala primjenom neuralnih mreža za sustave D-CDMA s preklapanjem snopa. Prikazana je metodologija procjene smjera dolaska signala željenog pokretnog korisnika uz primjenu neuralnih mreža za sustave s višestrukim pristupom po kodnoj osnovi s asinkronom izravnom sekvencijom (DS-CDMA). U sustavima DS-CDMA, valja procijeniti smjer dolaska signala željenog korisnika u nazočnosti više korisnika koji stvaraju interferencije (smetnje) što vodi do interferencije višestrukog pristupa. U ovom se radu rabi algoritam neuralnih mreža za potiskivanje interferencije uz primjenu kodno prilagođenih filtara kako bi se mogao odrediti smjer dolaska signala u sustavu s preklapanjem antenskog snopa. Razvijena je predložena metoda, a simulacijski pokusi s procjenom smjera dolaska signala pokazali su točne rezultate za različit broj pokretnih korisnika.

Ključne riječi: smjer dolaska, DS-CDMA, neuralne mreže, sustavi s preklapanjem snopa

\author{
AUTHORS' ADDRESS \\ Mr. Konstantinos A. Gotsis \\ Dr. Theodoros N. Kaifas \\ Assistant Professor Katherine Siakavara \\ Professor John N. Sahalos \\ Radiocommunications Lab Department of Physics \\ Aristotle University of Thessaloniki \\ 541 24, Thessaloniki \\ Greece
}

